\title{
Study of Relative Association, Apparent Molar Compressibility and Free Length of Entacapone API in alcohols at different Frequencies
}

\author{
Sachin Deorao Zade, Padma Sachin Zade, Pravin J. Ganjre, Sunanda Shashikant Aswale and \\ Shashikant Rajeshwar Aswale
}

Lokmanya Tilak Mahavidyalaya, Wani, Dist. Yavatmal, 445304 (MS).

\begin{abstract}
The values of ultrasonic velocity is measured by using ultrasonic interferometer for $0.01 \mathrm{M}$ solution of entacapone in methanol, ethanol, 1-propanol and 1-butanol as a solvent at $303.15 \mathrm{~K}$ by using frequency $2 \mathrm{MHz}, 4 \mathrm{MHz}$ and $6 \mathrm{MHz}$. This experimental data is used to explore the acoustic properties like intermolecular free length, relative association and apparent molar compressibility. From these thermodynamic and acoustic properties, the molecular interactions like solute-solute and solute-solvent occurring in solution have been interpreted with respect to change in solvent at different frequencies. It was observed that apparent molar compressibility value decreases from $2 \mathrm{MHz}$ to $6 \mathrm{MHz}$ in solvent Methanol to 1-butanol. Decreasing trend in Ultrasonic velocity shows less interaction between solute and solvent molecules. From $2 \mathrm{MHz}$ to $6 \mathrm{MHz}$ intermolecular free length increases which shows weak molecular interaction. Relative association increases from methanol to 1-butanol from increasing frequency i.e. from $2 \mathrm{MHz}$ to $6 \mathrm{MHz}$. It shows strong solute solvent interaction.
\end{abstract}

Key words: Ultrasonic interferometer, Apparent molar compressibility, Intermolecular free length, Relative association, Molecular interaction.

\section{INTRODUCTION}

Sound wave is useful in understanding physico-chemical behavior of liquid mixture. The solute-solvent molecular interaction was studied by Aswale S. S. et al. ${ }^{1-3}$ In view of the medicinal and pharmaceutical significance of Entacapone we intended to study the intermolecular interactions by ultrasonic interferometer. Ultrasonic velocity measurements are helpful to interpret solute-solvent, ion-solvent and solvent-solvent interaction in aqueous and non aqueous medium., ${ }^{4,5}$ Ultrasonic interferometer is non destructive technique which is particularly used for liquid mixture to determine molecular interactions in solute and solvent at different branches of science. According to Baskarana $\mathrm{R},{ }^{6}$ Herfeld $^{7}$ and Kannapan, ${ }^{8}$ the molecular interactions are mostly studied by ultrasonic interferometer. C.Rambabu ${ }^{9}$ studied ultrasonic velocity and density of binary liquid mixture, ultrasonic velocity measurements can be employed to detect and assess weak and strong molecular interactions present in all type of liquid mixtures (binary and ternary) has been studied by Ajitha S, Hema Malini A and Meena Devi VN, ${ }^{10}$ Neelam Shaky, Neha Shakya and R. S. Gangwar ${ }^{11}$ explain about specific acoustic impedance is the complex ratio of the effective sound pressure at a point to the effective particle velocity at that point to effective particle at that point.

In recent years measurement of ultrasonic investigation find extensive application in determining the physiochemical behavior of liquid mixture. To study the liquid
Submission Date : 05-07-2016

Revision Date : 31-08-2016

Accepted Date : :21-09-2016

DOI: 10.5530/ijper.50.4.23

Correspondence:

Sachin Deorao Zade,

Flat no 508, Building C, Aspiria Sociaty, Opp. Shell

Petrol Pump, Hinjawadi,

Pune-411057 (Maharastra)

INDIA.

Phone no. 9823503723

E-mail: sachind1981@gmail. com

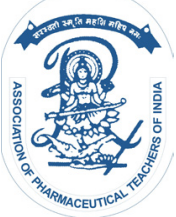

www.ijper.org 
theory, the acoustic properties such as ultrasonic velocity, apparent molar compressibility and relative association are very useful.

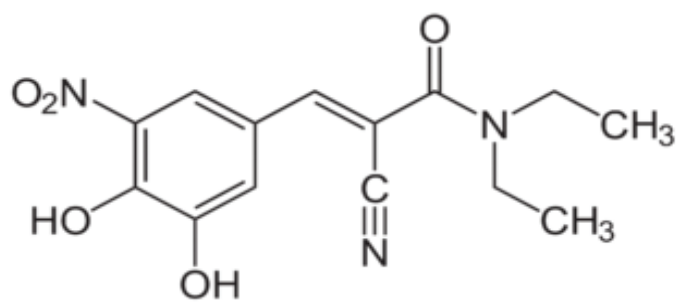

(2E)-2-cyano-3-(3,4-dihydroxy-5-nitrophenyl)-N,Ndiethylprop-2-enamide [Entacapone, M. W. 305.286]

No work has been reported on the ultrasonic studies of Entacapone drug substance on different frequencies in different alcohols, which will provide the information about molecular interactions between different solvents at different frequencies in the presence of solute Entacapone. Therefore the present work is undertaken to study the acoustical behavior of Entacapone drug substance in $0.01 \mathrm{M}$ concentration in different solvents to discuss the interactions of unlike molecule of solvents in presence of solute. Hence an attempt in this investigation is made to study the intermolecular interactions of Entacapone in methanol, ethanol, 1-propanol and 1-butanol at different frequencies like $2 \mathrm{MHz}$, $4 \mathrm{MHz}$ and $6 \mathrm{MHz}$, keeping the temperature constant i.e $303.15 \mathrm{~K}$ having concentration $0.01 \mathrm{M}$. The acoustic properties like apparent molar compressibility, relative association, inter molecular free length etc. are determined from the ultrasonic velocity and density measurements of the solution. From the observations of these properties the molecular interactions between solute, solvent are predicted.

\section{Experimental}

For the experimental work, Ramdev Chemicals provide Entacapone API as a gift sample. Advanced electronic densitometer Anton Paar-35 was used to measure the density having accuracy of $\pm 0.1 \mathrm{~kg} / \mathrm{m} 3$ which directly shows the temperature and density of solutions. Multi frequency interferometer (Mittal Enterprises, Model F-83) with accuracy of $\pm 0.03 \%$ at frequency 2,4 and 6 $\mathrm{MHz}$ was used in the present work.

AR grade methanol, ethanol, 1-propanol and 1-butanol were used as a solvent and density were measured at $303.15 \mathrm{~K}$. Weighing of sample was done on digital balance Model CB/CA/CT-Series, Contech, having accuracy $0.0001 \mathrm{~g}$. Calibration of instrument was done by measuring the ultrasonic velocity of pure water at $303.15 \mathrm{~K}$. The measured value $1511 \mathrm{~ms}^{-1}$ is very close to theoretical ${ }^{12}$ value $1515 \mathrm{~ms}^{-1}$ and thus confirms the accuracy of instrument. A $0.01 \mathrm{M}$ solution of Entacapone was prepared in methanol, ethanol, 1-propanol and 1-butanol separately. The densities and ultrasonic velocities of all solutions were measured at $303.15 \mathrm{~K}$. Same measurements were carried out in all four solvents methanol, ethanol, 1-propanol and 1-butanol for $0.01 \mathrm{M}$ solutions of Entacapone API.

\section{RESULTS AND DISCUSSION}

In the present investigation, measurements of densities and ultrasonic velocity of Entacapone API in solvent methanol, ethanol,1-propanol,1-butanol having concentration $0.01 \mathrm{M}$ at $303.15 \mathrm{~K}$ at $2 \mathrm{MHz}, 4 \mathrm{MHz}$ and 6 $\mathrm{MHz}$ frequency have been made, and given in Table 1.

Apparent molar compressibility $(\phi K)$ is an acoustic property which is determined by measuring density and ultrasonic velocity and depends upon the molality of solution and molecular weight of the solute, by the relation,

$$
\phi K=\left(1000 x \frac{\left(\beta_{s} \rho_{0}-\beta_{0} \rho_{s}\right)}{m \rho_{s} \rho_{0}}\right)+\left(\beta_{s} \frac{M}{\rho_{s}}\right)
$$

where, $\rho_{0}=$ density of pure solvent, $\rho_{\mathrm{s}}=$ density of solution, $\mathrm{m}=$ molality of solution, $\mathrm{M}=$ molecular weight of solute, $\beta_{0}=$ adiabatic compressibility of pure solvent and $\beta s=$ adiabatic compressibility of solution.

Relative association is a function of ultrasonic velocity and is computed by the equation,

$$
\mathrm{R}_{\mathrm{A}}=\frac{\rho_{\mathrm{s}}}{\rho_{0}}\left(\frac{\mathrm{v}_{0}}{\mathrm{v}_{\mathrm{s}}}\right)^{1 / 3}
$$

Where, $\mathrm{v}_{0}$ and $\mathrm{v}_{\mathrm{s}}$ are ultrasonic velocities in solvent and solution.

Intermolecular free length $(\mathrm{Lf})$ is one of the important acoustic properties to study the intermolecular interactions. According to Hildebrand ${ }^{13}$ the center of attraction does not coincide with geometrical center of molecule of liquid as the distance between the centers of attraction is a property extremely difficult to define. The distance between the surfaces of molecules, on the other hand has a clear physical significance so it is used in defining free length.

Intermolecular free length has been evaluated from adiabatic compressibility $(\beta)$ by Jacobson's ${ }^{14-16}$ formula,

$$
\mathrm{L}_{\mathrm{f}}=\mathrm{K} \cdot \sqrt{\beta_{\mathrm{s}}}
$$

From experimental data of velocity and density of solutions the thermodynamic and acoustic properties like 


\begin{tabular}{|c|c|c|c|c|c|c|c|}
\hline MHz & Solvents & $\begin{array}{l}\text { Density of } \\
\text { Solution } \\
\left(\mathrm{Kg} / \mathrm{m}^{3}\right)\end{array}$ & $\begin{array}{l}\text { Ultrasonic } \\
\text { velocity of } \\
\text { Solution } \\
\mathrm{m} / \mathrm{s}\end{array}$ & $\begin{array}{c}\text { Apparent Molar } \\
\text { Compressibility } \\
\text { of Solution }\end{array}$ & $\begin{array}{c}\text { Adiabatic } \\
\text { compressibility of } \\
\text { pure solvent }\end{array}$ & $\begin{array}{c}\text { Relative } \\
\text { Association }\end{array}$ & $\begin{array}{l}\text { Intermolecular } \\
\text { Free length }\end{array}$ \\
\hline \multirow{4}{*}{2} & Methanol & 795.7 & 5195.17 & $5.86 \mathrm{E}^{-09}$ & $6.13 \mathrm{E}^{-011}$ & $9.71 \mathrm{E}^{-01}$ & $4.31 \mathrm{E}^{-03}$ \\
\hline & Ethanol & 782.8 & 4271.01 & $8.96 \mathrm{E}^{-09}$ & $7.16 \mathrm{E}^{-011}$ & $9.97 \mathrm{E}^{-01}$ & $5.28 \mathrm{E}^{-03}$ \\
\hline & 1-propanol & 800.1 & 3330.19 & $1.41 \mathrm{E}^{-08}$ & $6.85 \mathrm{E}^{-011}$ & $1.09 \mathrm{E}^{+00}$ & $6.70 \mathrm{E}^{-03}$ \\
\hline & 1-butanol & 813.2 & 3181.55 & $1.50 \mathrm{E}^{-08}$ & $8.47 \mathrm{E}^{-011}$ & $1.08 \mathrm{E}^{+00}$ & $6.95 \mathrm{E}^{-03}$ \\
\hline \multirow{4}{*}{4} & Methanol & 795.7 & 9043.30 & $1.94 \mathrm{E}^{-09}$ & $1.66 \mathrm{E}^{-011}$ & $1.00 \mathrm{E}^{+00}$ & $2.47 \mathrm{E}^{-03}$ \\
\hline & Ethanol & 782.8 & 10003.02 & 1.63E-09 & $1.31 \mathrm{E}^{-011}$ & $9.96 \mathrm{E}^{-01}$ & $2.25 \mathrm{E}^{-03}$ \\
\hline & 1-propanol & 800.1 & 9104.26 & $1.89 \mathrm{E}^{-09}$ & $2.27 \mathrm{E}^{-011}$ & $9.40 \mathrm{E}^{-01}$ & $2.45 \mathrm{E}^{-03}$ \\
\hline & 1-butanol & 813.2 & 8383.04 & $2.16 \mathrm{E}^{-09}$ & $1.70 \mathrm{E}^{-011}$ & $1.02 \mathrm{E}^{+00}$ & $2.64 \mathrm{E}^{-03}$ \\
\hline \multirow{4}{*}{6} & Methanol & 795.7 & 11239.70 & $1.25 \mathrm{E}^{-09}$ & $1.49 E^{-011}$ & $9.51 \mathrm{E}^{-01}$ & $1.99 \mathrm{E}^{-03}$ \\
\hline & Ethanol & 782.8 & 8374.80 & $2.33 \mathrm{E}^{-09}$ & $1.10 \mathrm{E}^{-011}$ & $1.09 \mathrm{E}^{+00}$ & $2.69 \mathrm{E}^{-03}$ \\
\hline & 1-propanol & 800.1 & 7991.60 & $2.45 \mathrm{E}^{-09}$ & $4.67 \mathrm{E}^{-012}$ & $1.28 \mathrm{E}^{+00}$ & $2.79 \mathrm{E}^{-03}$ \\
\hline & 1-butanol & 813.2 & 14020.73 & $7.71 \mathrm{E}^{-10}$ & $5.80 \mathrm{E}^{-012}$ & $1.03 \mathrm{E}^{+00}$ & $1.58 \mathrm{E}^{-03}$ \\
\hline
\end{tabular}

apparent molar compressibility $(\phi K)$, Intermolecular free length $\left(\mathrm{L}_{\mathrm{f}}\right)$ and Relative association $(\mathrm{RA})$ are computed and results are tabulated in Table 1.

These acoustic parameters are directly reflecting the structural interaction of solvents with solute and explore valuable and important information regarding internal structure and molecular association.

By looking towards structures of all solvents it was observed that methylene group increases from methanol to 1-butanol, the molecules became more bulky, making the medium to be denser, this leads to greater compressibility resulting in slow transfer of sound waves and hence ultrasonic velocity decreases with increase of methylene group i.e from methanol to 1-butanol as shown in Figure 1. This decrease in Ultrasonic velocity shows less interaction between solute and solvent molecules. It is due to formation of less hydrogen bonding but at $6 \mathrm{MHz}$ the same sequence is obtained except 1-butanol which increases may be due to steric effect of larger butyl group.

The increase in ultrasonic velocity brings molecules to a closer packing which attributed strong intermolecular association results formation of $\mathrm{H}$-bonds between solute and solvent molecules.

Apparent molar compressibility $(\phi K)$ is important acoustic parameter, which explains the solute-solvent and solute-solute interactions in solutions.

It is not only depends upon ultrasonic velocity and density like $\beta$ but also the molecular weight of solute and molarity of solution. Thus, the structure of solute will have direct effect on $\phi K$ values. Apparent molar compressibility property is fairly sensitive to structure changes especially in highly structured solvent and is

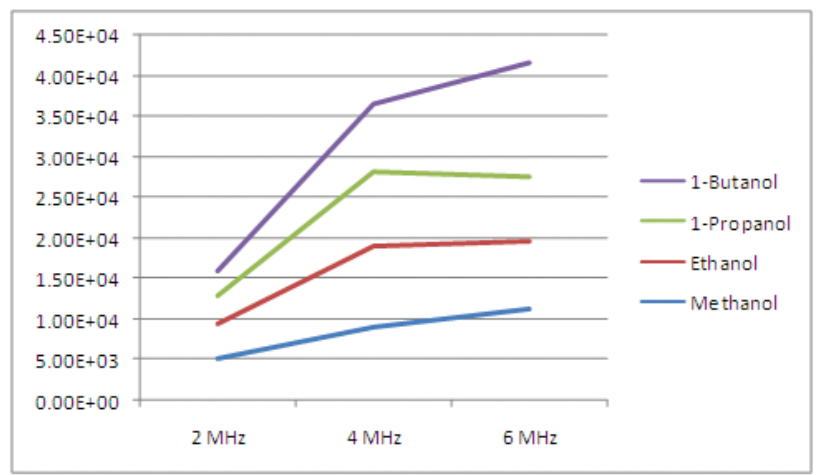

Figure 1: Ultrasonic Velocity of Entacapone at 0.01M \& 303.15K

hence expected to throw interesting light. From Figure 2 it was observed that apparent molar compressibility decreases from methanol to ethanol. But in 1-propanol and 1-butanol it is increases at $2 \mathrm{MHz}$. This trend also observed at $4 \mathrm{MHz}$ and $6 \mathrm{MHz}$. From the trend it was concluded that there is minimum difference between values of apparent molar compressibility on $2 \mathrm{MHz}$, $4 \mathrm{MHz}$ and $6 \mathrm{MHz}$. This observed difference is may be because of linkage of methylene group in different solvents. Here there are clear differences observed between two properties i.e. adiabatic compressibility and apparent molar compressibility. This difference may be because of freely movement of entacapone sample ions in the solution which is prepared in different solvents.

Relative association is an acoustic property of understanding interaction, which is influenced ${ }^{17-18}$ by two opposing factors:

1. Breaking of solvent structure on addition of solute to it and 


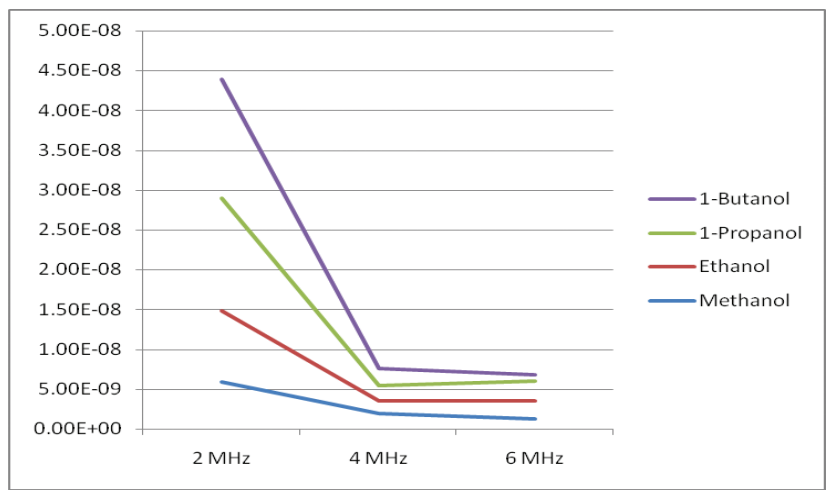

Figure 2: Apparent molar Compressibility of Entacapone at $0.01 \mathrm{M} \& 303.15 \mathrm{~K}$

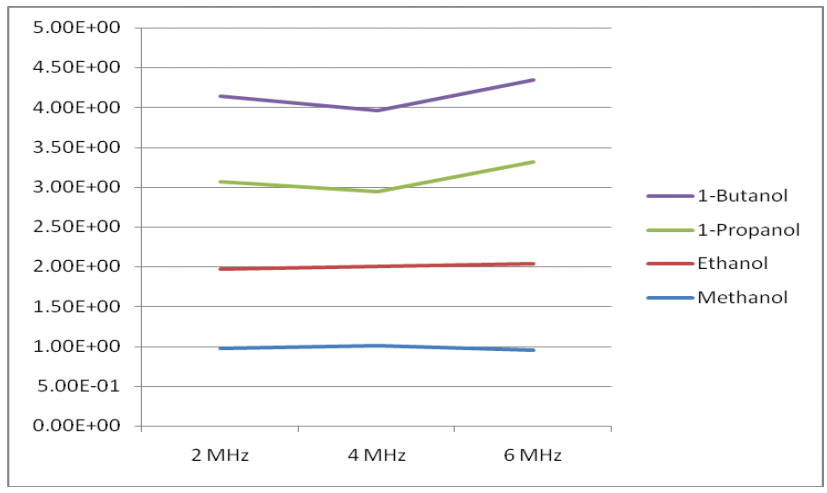

Figure 3: Relative Association of Entacapone at 0.01M \& 303.15K

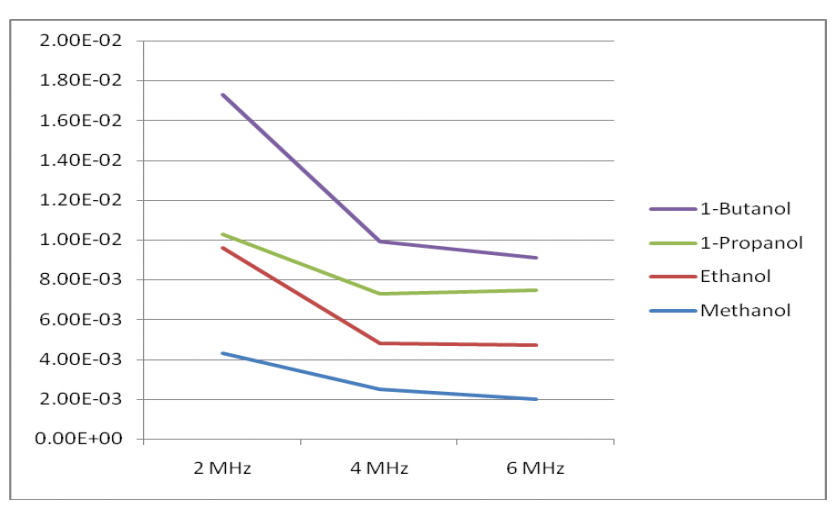

Figure 4: Intermolecular free length of Entacapone at $0.01 \mathrm{M}$ \& $303.15 \mathrm{~K}$

2. Salvation of the solutes, those are simultaneously present, by the free solvent molecule.

Relative association as seen in Figure 3 varies non linearly with change in solvent which reveals that the specific interaction is existing in the solution. Relative association is the measure of extent of association of components in the medium. It is a property of understanding the molecular interaction in liquid mixtures and solutions. As discussed relative association depends on either of breaking up of the solvent molecules on addition of solute to it or the salvation of ions that are present. From Figure 3 it was observed that relative association $\left(\mathrm{R}_{\mathrm{A}}\right)$ increases from methanol to 1-butanol from increasing frequency i.e. from $2 \mathrm{MHz}$ to $6 \mathrm{MHz}$. It shows strong solute solvent interaction. After evaluation of structures of all solvents it was observed that there is chain of methylene group. This increasing trend of $R_{A}$ may be because of strong hydrogen bonding happening because of presence of methylene group.

Intermolecular free length is the distance between surfaces of the neighboring molecules. Eyring and Kincaid ${ }^{19}$ have proposed that free length is dominant factor in determining the variation of ultrasonic velocity of solution. Ultrasonic velocity depends on intermolecular free length Lf, with decrease in free length velocity increases or vice versa. Mathematically intermolecular free length is inversely proportional to ultrasonic velocity and directly proportional to adiabatic compressibility shows similar behavior to that of adiabatic compressibility and opposite to that of Ultrasonic velocity. As per Figure 4 free length increases from solvent methanol to 1-butanol as frequency increase from $2 \mathrm{MHz}, 4 \mathrm{MHz}$ and $6 \mathrm{MHz}$ it is because of weak molecular interaction. While after evaluating structures of all four solvents, it was observed that if chain of methylene group and entacapone contains electron withdrawing group which increases close packing in the solution and hence free length decrease. But the strong dipole-dipole interaction exists at $6 \mathrm{MHz}$ in 1-butanol which resulting in low value of free length. Low value of free length in 1-butanol solution indicates that strong interaction between solute and solvent suggesting structure promoting behavior of solute.

As free length increases with increase in frequencies, shows weak molecular interaction while decrease in free length signifies specific interaction existing in the solution.

\section{CONCLUSION}

In present investigation, physical and acoustic parameters were determined for entacapone in solvents like Methanol, Ethanol, 1-propanal and 1-butanol at 2, 4 and $6 \mathrm{MHz}$ by using constant temperature i.e. $303.15 \mathrm{~K}$. From Table 1 it was observed that, apparent molar compressibility decreases from methanol to ethanol. But in 1-propanol and 1-butanol it is increases at $2 \mathrm{MHz}, 4 \mathrm{MHz}$ and $6 \mathrm{MHz}$. When methylene group introduced into the aromatic ring then it causes to change in $\pi$ electron density around the aromatic ring. This is because of positive mesomeric and positive electrometric effect. This observed difference is may be because of linkage of methylene group in different solvents. 
Also it was observed that, free length decreases from solvent methanol to 1-butanol as frequency increase from $2 \mathrm{MHz}, 4 \mathrm{MHz}$ and $6 \mathrm{MHz}$ it is because of weak molecular interaction. There is chain of methylene group and entacapone contains electron withdrawing group which increases close packing in the solution and hence free length decrease.

Relative association $\left(R_{A}\right)$ increases from methanol to 1-butanol from increasing frequency i.e. from $2 \mathrm{MHz}$ to $6 \mathrm{MHz}$. It shows strong solute solvent interaction. After evaluation of structures of all solvents it was observed that there is chain of methylene group. This increasing trend of $\mathrm{R}_{\mathrm{A}}$ may be because of strong hydrogen bonding happening in the solution because of presence of methylene group. This increasing trend of relative association value shows stronger interaction between solute and solvent.

\section{ACKNOWLEDGEMENT}

I wish to express my sincere gratitude to Dr. Aparna Dhote and Dr. Rajesh Hajare as they provided valuable literature and information during this research work.

\section{CONFLICT OF INTEREST}

Dr. Aparna Dhote and Dr. Rajesh Hajare are Senior researcher's who gave valuable advice.

\section{ABBREVIATION USED}

$\rho \mathbf{0}$ : Density of pure solvent; $\rho$ s: Density of solution; $\mathrm{m}$ : Molality of solution; M: Molecular weight of solute; $\beta 0$ : Adiabatic compressibility of pure solvent; $\beta$ s: Adiabatic compressibility of solution; $\phi \mathbf{K}$ : Apparent molar compressibility; $\mathbf{R}_{\mathbf{A}}$ : Relative association; v0: Ultrasonic velocities of solvent; vs: Ultrasonic velocities of solution; Lf: Intermolecular free length; $\beta$ : Adiabatic compressibility.

\section{REFERENCES}

1. Aswale SS, Raghuwanshi PB, Tayade DT, Aswale SR, J Indian Chem Soc. 2007;84(2);159-64.

2. Aswale SS, Aswale SR, Tayade DT, Raghuwanshi PB. Proceedings of 1st International Society Bio-Technology Conference-2008, Gangtok, 2008;325.

3. Aswale SS, Aswale SR, Tayade DT, Raghuwanshi PB. J Pure and Appl. Ultrason. 2008;30:62-8.

4. Tabhane VA. Acoust Lett (G.B). 1983;6:120.

5. Balaja S, Oza S. 2012;200:11-8.

6. Baskarana R, Kubendran PR. Int J Appl Sci and Engg. 2007;5:115.

7. Herfeld KF. Litovitz, "Absorption and dispersion of ultrasonic waves", Academic Press, New York, (1959).

8. Kannapan V, Jayashanti R. Indian J Chem. 43(A)(2004), 1431.

9. Rambabu C. Chemical Science Transitions. 2015;4(1):17-26.

10. Ajitha S, Hema Malini A, Meena Devi VN. July-September. 2013;4;(3):224

11. Neelam S, Neha S, Gangwar RS. Solvoytic study of binary mixtures of iron pentacarbonyl in chlorobenzene at 350C, Rasayan . J Chem. 2011;4(2):442.

12. Hall L. "The Origin of Ultrasonic Absorption in Water",. Phys Rev. 1998;73(5):775

13. Hildebrand JH. "The Solubility of Non-electrolytes", Ed. 3, Rein Hold Publishing Corp., New York, 1950, 48.

14. Jocobson B, Acta. Chem. Scand. 1952;8:1485-95. http://dx.doi.org/10.3891/ acta.chem.scand.06-1485

15. Jacobson B. J Chem Phys. 1952;1;20(5):927-8.

16. Jacobson B. Acta. Chem. Scand. 1951;5(7-8):1214-6. http://dx.doi. org/10.3891/acta.chem.scand.05-1214

17. Baluja S. J Indian Chem Soc. 2004;81(7):570.

18. Kiyohara O, Benson GC. J Chem. Thermodyn. 1979;11(9):861-73. http:// dx.doi.org/10.1016/0021-9614(79)90067-3

19. Eyring B, Kincaid JF. J Chem Phys. 1938;6(10):620-9. http://dx.doi. org/10.1063/1.1750134

\section{SUMMARY}

- A decrease in adiabatic compressibility is may be because of positive mesomeric effect and positive electromagnetic effect.

- Increasing trend of Relative association is may be because of strong hydrogen bonding happening in the solution because of presence of methylene group. 